\title{
First detection of ND in the solar-mass protostar IRAS16293-2422 ${ }^{\star}$
}

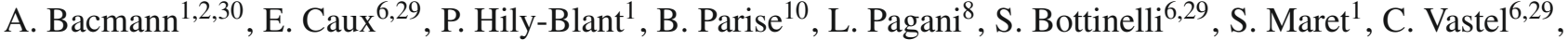 \\ C. Ceccarelli ${ }^{1,2,30}$, J. Cernicharo ${ }^{9}$, T. Henning ${ }^{26}$, A. Castets ${ }^{1}$, A. Coutens ${ }^{6,29}$, E. A. Bergin ${ }^{25}$, G. A. Blake ${ }^{3}$, \\ N. Crimier ${ }^{1,9}$, K. Demyk ${ }^{6,29}$, C. Dominik ${ }^{12,13}$, M. Gerin ${ }^{28}$, P. Hennebelle ${ }^{28}$, C. Kahane ${ }^{1}$, A. Klotz ${ }^{6,29}$, G. Melnick ${ }^{18}$, \\ P. Schilke ${ }^{10,20}$, V. Wakelam ${ }^{2,30}$, A. Walters ${ }^{6,29}$, A. Baudry ${ }^{2,30}$, T. Bell ${ }^{3}$, M. Benedettini ${ }^{4}$, A. Boogert ${ }^{5}$, S. Cabrit ${ }^{8}$, \\ P. Caselli ${ }^{7}$, C. Codella ${ }^{11}$, C. Comito ${ }^{10}$, P. Encrenaz ${ }^{8}$, E. Falgarone ${ }^{28}$, A. Fuente ${ }^{14}$, P. F. Goldsmith ${ }^{15}$, F. Helmich ${ }^{16}$,

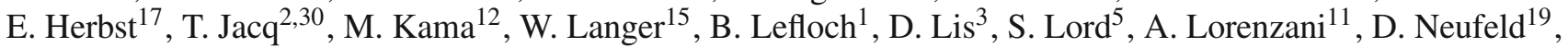 \\ B. Nisini ${ }^{24}$, S. Pacheco ${ }^{1}$, J. Pearson ${ }^{15}$, T. Phillips ${ }^{3}$, M. Salez ${ }^{8,28}$, P. Saraceno ${ }^{4}$, K. Schuster ${ }^{21}$, X. Tielens ${ }^{22}$, \\ F. F. S. van der Tak ${ }^{16,27}$, M. H. D. van der Wiel ${ }^{16,27}$, S. Viti ${ }^{23}$, F. Wyrowski ${ }^{10}$, H. Yorke ${ }^{15}$, A. Faure ${ }^{1}$, A. Benz ${ }^{31}$, \\ O. Coeur-Joly ${ }^{6,29}$, A. Cros ${ }^{6,29}$, R. Güsten ${ }^{10}$, and L. Ravera ${ }^{6,29}$
}

(Affiliations are available on page 5 of the online edition)

Received 31 May 2010 / Accepted 30 June 2010

\begin{abstract}
Context. In the past decade, much progress has been made in characterising the processes leading to the enhanced deuterium fractionation observed in the ISM and in particular in the cold, dense parts of star forming regions such as protostellar envelopes. Very high molecular D/H ratios have been found for saturated molecules and ions. However, little is known about the deuterium fractionation in radicals, even though simple radicals often represent an intermediate stage in the formation of more complex, saturated molecules. The imidogen radical NH is such an intermediate species for the ammonia synthesis in the gas phase. Many of these light molecules however have their fundamental transitions in the submillimetre domain and their detection is hampered by the opacity of the atmosphere at these wavelengths. Herschel/HIFI represents a unique opportunity to study the deuteration and formation mechanisms of species not observable from the ground.

Aims. We searched here for the deuterated radical ND in order to determine the deuterium fractionation of imidogen and constrain the deuteration mechanism of this species.

Methods. We observed the solar-mass Class 0 protostar IRAS16293-2422 with the heterodyne instrument HIFI in Bands 1a (480-560 GHz), 3b (858-961 GHz), and 4a (949-1061 GHz) as part of the Herschel key programme CHESS (Chemical HErschel Survey of Star forming regions). Results. The deuterated form of the imidogen radical ND was detected and securely identified with 2 hyperfine component groups of its fundamental transition $(N=0-1)$ at 522.1 and $546.2 \mathrm{GHz}$, in absorption against the continuum background emitted from the nascent protostar. The 3 groups of hyperfine components of its hydrogenated counterpart $\mathrm{NH}$ were also detected in absorption. The absorption arises from the cold envelope, where many deuterated species have been shown to be abundant. The estimated column densities are $\sim 2 \times 10^{14} \mathrm{~cm}^{-2}$ for $\mathrm{NH}$ and $\sim 1.3 \times 10^{14} \mathrm{~cm}^{-2}$ for ND. We derive a very high deuterium fractionation with an $[\mathrm{ND}] /[\mathrm{NH}]$ ratio of between 30 and $100 \%$.

Conclusions. The deuterium fractionation of imidogen is of the same order of magnitude as that in other molecules, which suggests that an efficient deuterium fractionation mechanism is at play. We discuss two possible formation pathways for ND, by means of either the reaction of $\mathrm{N}^{+}$ with $\mathrm{HD}$, or deuteron/proton exchange with $\mathrm{NH}$.
\end{abstract}

Key words. ISM: molecules - stars: formation

\section{Introduction}

The envelopes of low-mass Class 0 protostars have been known to be characterised by high levels of molecular deuteration, as shown e.g., by Loinard et al. (2002), Roberts et al. (2007, 2002), and the detection of multiply deuterated species (Ceccarelli et al. 1998 for $\mathrm{D}_{2} \mathrm{CO}$, Parise et al. 2002 for $\mathrm{CHD}_{2} \mathrm{OH}$, Parise et al. 2004 for $\mathrm{CD}_{3} \mathrm{OH}$, van der Tak et al. 2002 for $\mathrm{ND}_{3}$ ). The high abundance of deuterated molecules is believed to originate in the pre-stellar phase (Bacmann et al. 2003): at the low temperatures $(<20 \mathrm{~K})$ and high densities $\left(n>10^{4} \mathrm{~cm}^{3}\right)$ prevailing in pre-stellar cores (and similarly in protostellar envelopes), heavy molecules such as $\mathrm{CO}$ are depleted on the dust grains, so that

* Herschel is an ESA space observatory with science instruments provided by European-led principal Investigator consortia and with important participation from NASA. species such as $\mathrm{H}_{2} \mathrm{D}^{+}, \mathrm{D}_{2} \mathrm{H}^{+}$, or $\mathrm{D}_{3}^{+}$reach high abundances. The increase in the $\left[\mathrm{H}_{2} \mathrm{D}^{+}\right] /\left[\mathrm{H}_{3}^{+}\right]$ratio (and similar ratios involving multiply deuterated counterparts) enhances both the molecular deuterium fractionation by means of gas-phase reactions and the atomic $\mathrm{D} / \mathrm{H}$ ratio, therefore also increasing the deuteration of species forming on grain surfaces (e.g. Roberts et al. 2003).

Most deuterated molecules detected to date are either saturated molecules or ions. The deuteration of radicals, which are important intermediate pieces leading to the formation of saturated molecules, has so far not been thoroughly investigated. Their observation could however provide an important clue to the formation process of the major deuterated species. For example, it is not yet completely understood if saturated deuterated molecules are formed directly (from deuterated ion intermediates, whose dissociative recombination may lead to deuterated radicals) like their hydrogenated counterparts, or if 
they are formed by proton-deuteron exchange starting from the saturated hydrogenated molecule. The observation of the $\mathrm{D} / \mathrm{H}$ ratio in the radical may help us to disentangle these two processes. With this in mind, we study the deuteration ratio in the $\mathrm{NH}$ radical, which is formed from the dissociative recombination of $\mathrm{NH}_{2}^{+}$and $\mathrm{NH}_{3}^{+}$, two precursors of ammonia in the gas-phase. Roueff et al. (2005) argued that highly-deuterated ammonia can form from pure gas phase reactions. These models however require highly deuterated radicals, and we propose to observationally test this model.

Light hydrides such as NH have fundamental transitions in the submillimetre domain: $\mathrm{NH}$ possesses 3 groups of hyperfine transitions around $950 \mathrm{GHz}-1 \mathrm{THz}$ and its deuterated counterpart ND has its 3 groups of fundamental hyperfine structure transitions around $490-550 \mathrm{GHz}$. At all of these frequencies apart from $490 \mathrm{GHz}$, the atmosphere is opaque and precludes observations from the ground. However, these transitions can all be observed with the HIFI instrument on board the Herschel Space Observatory. Since hydrides generally have high Einstein $A_{\mathrm{ul}}$ coefficients, only the ground state is expected to be significantly populated. As a consequence, it is possible to detect these species in absorption against the continuum background originating from the heating of the nascent protostar. Moreover, as already mentioned above, deuterated molecules are very abundant in cold, dense medium, so that the envelopes of young protostars represent ideal targets to study the deuteration of the $\mathrm{NH}$ radical.

In the course of the CHESS key programme (Ceccarelli et al. 2010), we carried out a spectral survey of the frequencies covered by HIFI in the young low-mass protostar IRAS 16293-2422 (hereafter IRAS16293). The frequency bands contain the fundamental hyperfine transitions of ND and NH. In this Letter, we report the first detection by HIFI of ND, as well as the detection of the hyperfine transitions of NH. Section 2 describes the observations and the determination of the radicals' column densities, and Sect. 3 discusses the implications of the derived deuterium fractionation on our understanding of deuteration processes in N-bearing species.

\section{Observations and results}

The solar-mass protostar IRAS16293 was observed with the HIFI instrument (de Grauuw et al. 2010; Roelfsema et al. 2010) on board the Herschel Space Observatory (Pilbratt et al., 2010), as part of the HIFI guaranteed time key programme CHESS (Ceccarelli et al. 2010). A full spectral coverage of bands 1a (480-560 GHz), 3b (858-961 GHz), and 4a $(949-1061 \mathrm{GHz})$ was performed on 2010 March 1, 19, and 3, respectively, using the HIFI spectral scan double beam switch (DBS) mode with optimisation of the continuum. In this mode, the HIFI acousto-optic wide band spectrometer (WBS) was used, providing a spectral resolution of $1.1 \mathrm{MHz}\left(\sim 0.6 \mathrm{~km} \mathrm{~s}^{-1}\right.$ at $520 \mathrm{GHz}$ and $0.3 \mathrm{~km} \mathrm{~s}^{-1}$ at $1 \mathrm{THz}$ ) over an instantaneous bandwidth of $4 \times 1 \mathrm{GHz}$. The targeted coordinates were $\alpha_{2000}=16^{\mathrm{h}} 32^{\mathrm{m}} 22^{\mathrm{s}} .75$, $\delta_{2000}=-24^{\circ} 28^{\prime} 34.2^{\prime \prime}$. The DBS reference positions were situated approximately $3^{\prime}$ east and west of the source. The beam sizes at the frequencies of the ND and $\mathrm{NH}$ transitions are about $41^{\prime \prime}$ and $22^{\prime \prime}$, respectively, and the theoretical main beam and forward efficiencies are about 0.72 and 0.96 , respectively. Calibration uncertainties are $\leq 16 \%$ for band 1 and $\leq 32 \%$ for bands 3 and 4 (June 2010 estimates).

The data were processed using the standard HIFI pipeline up to frequency and amplitude calibrations (level 2) with the ESA-supported package HIPE 2.8 (Ott et al. 2010). A single local oscillator tuning spectrum consists, for each polarisation, of
4 sub-bands of $\sim 1 \mathrm{GHz}$ for the SIS bands (1 to 5). The $1 \mathrm{GHz}$ scans are then exported as FITS files into CLASS/GILDAS format $^{1}$ prior to data reduction and analysis using generic spectral survey tools developed in CLASS by our group. Spurious features in the spectra were first removed in each $1 \mathrm{GHz}$ scan, and a low order (typically 3) polynomial baseline was then fitted over line-free regions to correct for residual bandpass effects. These polynomials were subtracted and used to determine an accurate continuum level by calculating their medians. Higher order oscillations in the baseline are possible, but their amplitudes remain low in the spectra considered here. The continuum values obtained in this way are well fitted by a degree 1 polynomial over the frequency range $\sim 500-1200 \mathrm{GHz}$. Sideband deconvolution is computed with the minimisation algorithm of Comito \& Schilke (2002) implemented into CLASS90 using the baselinesubtracted spectra. The single side-band continuum derived from the polynomial fit at the considered frequency (Table 1) was eventually added to the spectra. The temperatures were converted to the $T_{\mathrm{mb}}$ scale, using the theoretical values of the main beam and forward efficiencies given above.

We detect for the first time the 3 groups of hyperfine transitions of ND at 491.9, 522.1, and 546.2 GHz of the $N=0-1$ transition as well as the 3 groups of hyperfine components of the NH fundamental transitions at 946.5, 974.5 GHz, and $1 \mathrm{THz}$ (see Klaus et al. 1997 for an energy diagram of the $N=0-1$ transition of $\mathrm{NH}$ ). The transitions are seen in absorption against the continuum from the protostar and the hyperfine structure is partially resolved. The transitions of ND at 522.1 and at $546.2 \mathrm{GHz}$ are presented in Fig. 1. From Fig. 1, the lines can be unambiguously attributed to ND (but see Olofsson et al. 2007, who tentatively assign an unidentified line to ND). The transition of ND at $491.9 \mathrm{GHz}$ is not shown here because some of its components are strongly blended with a bright $\mathrm{H}_{2} \mathrm{CO}$ line at $491.9683 \mathrm{GHz}$, with an $\mathrm{SO}_{2}$ line at $491.9347 \mathrm{GHz}$ and with an $\mathrm{HDCO}$ line at $491.9370 \mathrm{GHz}$. This transition was not used in our analysis and will not be discussed further. Figure 2 presents the 3 detected hyperfine groups of the fundamental transition for $\mathrm{NH}$.

The analysis was carried out by fitting the hyperfine structure using the HFS method in the CLASS software ${ }^{2}$. The line parameters (frequencies, Einstein $A_{\mathrm{ul}}$ coefficients, and level degeneracies), originally from spectroscopic studies of Klaus et al. (1997) and Saito \& Goto (1993) for NH and ND, respectively, were taken from the Cologne Database for Molecular Spectroscopy (Müller et al. 2001, 2005). The routine assumes a zero-baseline spectrum (i.e., from which the continuum emission has been subtracted) and therefore fits a line profile of the form $\left(1-\mathrm{e}^{-\tau}\right)\left(J_{v}\left(T_{\mathrm{ex}}\right)-J_{v}\left(T_{\mathrm{bg}}\right)-T_{\mathrm{c}}\right)$, where $\tau$ is the optical depth, $T_{\mathrm{c}}$ the intensity of the continuum, and $J_{v}\left(T_{\mathrm{ex}}\right)$ and $J_{v}\left(T_{\mathrm{bg}}\right)$ the radiation temperature for the excitation temperature $T_{\text {ex }}$ and the radiation temperature of the cosmological background, respectively. The parameters given by the HFS fitting procedure are reported in Table 2, Cols. (2) to (4). From the total optical depth $\tau$ given by the fit, we can infer the opacities of single hyperfine components by multiplying $\tau$ by the relative intensities of these components. The total column density of the considered species is given by

$$
N=\frac{8 \pi v^{3}}{c^{3}} \frac{Q\left(T_{\mathrm{ex}}\right)}{g_{\text {up }} A_{\mathrm{ul}}} \frac{\mathrm{e}^{\frac{E_{\mathrm{up}}}{k \mathrm{e}_{\mathrm{ex}}}}}{\mathrm{e}^{\frac{h \nu}{T_{\mathrm{ex}}}}-1} \int \tau \mathrm{d} v
$$

\footnotetext{
1 http://wWw . iram. fr/IRAMFR/GILDAS

2 see the CLASS manual at http://Www.iram.es/IRAMES/ otherDocuments/manuals/index.html for more details.
} 
Table 1. Measured rms (at a frequency resolution of 1.1 MHz) and assumed continuum values for the different transitions (in $T_{\mathrm{mb}}$ scale).

\begin{tabular}{ccccc}
\hline \hline HIFI band & $\begin{array}{c}\text { Transition } \\
\left(N_{J}-N^{\prime}{ }^{\prime}\right)\end{array}$ & $\begin{array}{c}\text { Frequency } \\
(\mathrm{GHz})\end{array}$ & $\begin{array}{c}\text { rms } \\
(\mathrm{mK})\end{array}$ & $\begin{array}{c}\text { Continuum } \\
(\mathrm{mK})\end{array}$ \\
\hline 1a & $\mathrm{ND}\left(0_{1}-1_{2}\right)$ & 522.1 & 12 & $203 \pm 22$ \\
1a & $\mathrm{ND}\left(0_{1}-1_{1}\right)$ & 546.2 & 15 & $230 \pm 24$ \\
3b & $\mathrm{NH}\left(0_{1}-1_{0}\right)$ & 946.5 & 58 & $832 \pm 80$ \\
4a & $\mathrm{NH}\left(0_{1}-1_{2}\right)$ & 974.5 & 49 & $892 \pm 143$ \\
4a & $\mathrm{NH}\left(0_{1}-1_{1}\right)$ & 1000.0 & 50 & $942 \pm 146$ \\
\hline
\end{tabular}

where $v$ is the frequency of the transition, $c$ the velocity of light, $g_{\text {up }}$ the upper level degeneracy, $A_{\mathrm{ul}}$ the spontaneous emission Einstein coefficient, $T_{\mathrm{ex}}$ the excitation temperature, $Q\left(T_{\mathrm{ex}}\right)$ the partition function at $T_{\mathrm{ex}}$, and $E_{\text {up }}$ the upper level energy. For the values of the excitation temperature and the frequencies considered here, the factor with the exponentials is nearly equal to 1 . The velocity integrated optical depth of component $i$ is given by $\tau_{i} \Delta v \frac{\sqrt{\pi}}{2 \sqrt{\ln 2}}$ with $\tau_{i}$ the optical depth of component $i$ and $\Delta v$ the linewidth determined by the HFS method (full width at half maximum). The obtained values of the column density are given for each group of hyperfine components in Col. (6) of Table 2. In our derivation of the excitation temperature, it was assumed that the zone where the absorption arises completely fills the emission zone of the continuum. In the HFS fitting method, the determination of the optical depth comes only from the measured relative ratios of the hyperfine components and is independent of any assumption about the filling factor. Overall, since the exponential factor is close to 1 in Eq. (1), and the partition function depends only mildly on $T_{\mathrm{ex}}$ (in the case of $\mathrm{NH}$ for example, it increases by $10 \%$ when $T_{\text {ex }}$ increases from 10 to $15 \mathrm{~K}$ ), the inferred value of the column density depends very little on the assumptions about the value of the continuum, beam efficiency values, or absolute calibration uncertainties.

The column density found for $\mathrm{NH}$ is $N(\mathrm{NH})=(2.0 \pm 0.8) \times$ $10^{14} \mathrm{~cm}^{-2}$. For ND, the column density values found from the 2 observed transitions differ slightly but are consistent within the error bars. A reasonable estimate for the ND column density is therefore: $N(\mathrm{ND})=(1.3 \pm 0.8) \times 10^{14} \mathrm{~cm}^{-2}$.

With these values, we find a imidogen deuterium fraction of $[\mathrm{ND}] /[\mathrm{NH}] \sim 30-100 \%$ in the envelope of IRAS16293. We note however that because of the differences in beam sizes, the region sampled by the ND observations is larger and on average characterised by lower densities and temperatures than the region sampled by the NH observations for which the contribution of the warmer inner regions is larger. To take these effects into account, a more detailed radiative transfer modelling - taking into account the source structure and the exact coupling between the source and the instrument - would be necessary. The similarity of the determined linewidths (around $0.5 \mathrm{~km} \mathrm{~s}^{-1}$ in both lines, when deconvolved from the resolution of the spectrometer) is consistent with the bulk of the signal from both molecules arising from the same (cold) region.

\section{Discussion and conclusions}

The very high deuterium fractionation observed in IRAS16293 is of the same order of magnitude as the molecular $\mathrm{D} / \mathrm{H}$ ratio previously measured in other species in the envelope of IRAS16293, i.e., $\left[\mathrm{HDCO} / \mathrm{H}_{2} \mathrm{CO}\right]=15 \%,\left[\mathrm{NH}_{2} \mathrm{D}\right] /\left[\mathrm{NH}_{3}\right]=$ $10 \%$ (van Dishoeck et al. 1995) $\left[\mathrm{CH}_{2} \mathrm{DOH}\right] /\left[\mathrm{CH}_{3} \mathrm{OH}\right]=30 \%$ (Parise et al. 2004). The $[\mathrm{ND}] /[\mathrm{NH}]$ ratio even appears to be the highest measured deuterium fractionation in this source, though this result needs to be confirmed with a more detailed modelling.

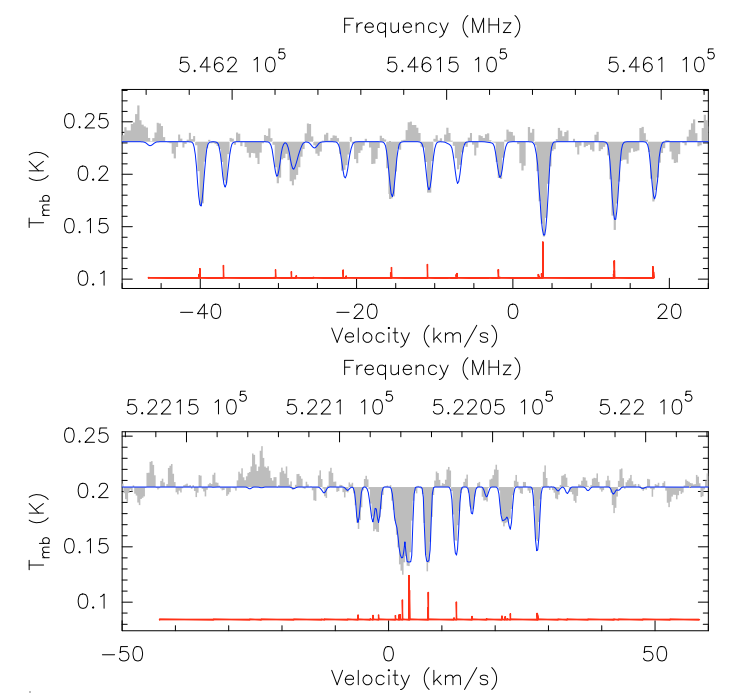

Fig. 1. $\mathrm{ND}\left(N_{J}=0_{1}-1_{1}\right)$ transition at $546.15 \mathrm{GHz}$ (upper panel) and $\mathrm{ND}\left(N_{J}=0_{1}-1_{2}\right)$ transition at $522.1 \mathrm{GHz}$ (lower panel). The filled histograms show the observed spectra and the solid line is the fit of the hyperfine structure performed using the CLASS software. At the bottom of each panel is a sketch of the positions and relative intensities of the hyperfine components. Components at the same frequency have been slightly put apart on the velocity axis for visualization reasons. The origin of the velocity axis was chosen so that the strongest component is at the LSR velocity of the source, $v=3.8 \mathrm{~km} \mathrm{~s}^{-1}$. The emission feature towards $522.12 \mathrm{GHz}$ coincides with a methanol line.

Our measured ratio is consistent with the gas-phase predictions of Roueff et al. (2005), although this model assumes that $\mathrm{NH}$ is a major product of the dissociative recombination of $\mathrm{N}_{2} \mathrm{H}^{+}$, a result that has since been refuted (Molek et al. 2007; Talbi 2009).

The main path for the gas-phase formation of $\mathrm{NH}$ in the dense interstellar medium is believed to be initiated by the endothermic $(85 \mathrm{~K})$ reaction $\mathrm{N}^{+}+\mathrm{H}_{2} \rightarrow \mathrm{NH}^{+}+\mathrm{H}$ (e.g., Galloway $\&$ Herbst 1989), which turns into $\mathrm{NH}_{2}^{+}, \mathrm{NH}_{3}^{+}$, and $\mathrm{NH}_{4}^{+}$after successive additions of $\mathrm{H}_{2}$. We then expect $\mathrm{NH}$ to be mostly a product of the dissociative recombination of electrons with $\mathrm{NH}_{2}^{+}$ and $\mathrm{NH}_{3}^{+}$(Thomas et al. 2005), while $\mathrm{NH}_{4}^{+}$leads to ammonia. One possible way of forming ND is by means of the reaction $\mathrm{N}^{+}$ $+\mathrm{HD} \rightarrow \mathrm{ND}^{+}+\mathrm{H}$. This reaction is less endothermic than the corresponding reaction with $\mathrm{H}_{2}$ (16 K - Marquette et al. 1988) so is more likely to occur at low temperatures. Furthermore, experiments indicate that the dissociative recombination of deuterated ions tends to eject $\mathrm{H}$ atoms preferentially (see Roueff et al. 2005 and references therein). The dissociative recombination of e.g. $\mathrm{NHD}^{+}$or $\mathrm{NH}_{2} \mathrm{D}^{+}$should therefore favour the production of ND with respect to $\mathrm{NH}$. On the other hand, the low abundance of HD $\left(\sim 3.2 \times 10^{-5}\left[\mathrm{H}_{2}\right]\right.$, Linsky 2007) might make it difficult to attain $[\mathrm{ND}] /[\mathrm{NH}]$ ratios around our measured value.

An alternative means of forming ND is by proton-deuteron reactions with $\mathrm{NH}$, i.e., $\mathrm{NH}+\mathrm{H}_{2} \mathrm{D}^{+} \rightarrow \mathrm{NHD}^{+}+\mathrm{H}_{2}$, followed by a dissociative recombination of $\mathrm{NHD}^{+}$, which leads predominantly to the deuterated molecule. This scheme is similar to that proposed by Rodgers \& Charnley (2001) to account for the high abundances of doubly deuterated ammonia, and relies on there being high abundances of species such as $\mathrm{H}_{2} \mathrm{D}^{+}$. In cold dense regions depleted of heavy elements such as $\mathrm{CO}$, the ratio of $\left[\mathrm{H}_{2} \mathrm{D}^{+}\right] /\left[\mathrm{H}_{3}^{+}\right]$is indeed highly enhanced (e.g. Caselli et al. 2008, 2003; Roberts et al. 2003). The ion $\mathrm{H}_{2} \mathrm{D}^{+}$has indeed been detected in the cold envelope of IRAS16293 by Stark et al. (2004), who estimated its abundance to be $2 \times 10^{-9}$. Although both routes of ND formation considered here seem plausible, it is not 
Table 2. Parameters given by the HFS fit of each ND and NH transition.

\begin{tabular}{|c|c|c|c|c|c|}
\hline $\begin{array}{l}\text { Molecule } \\
\& \text { transition }\end{array}$ & 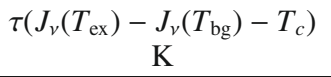 & $\begin{array}{c}\Delta v \\
\mathrm{~km} \mathrm{~s}^{-1}\end{array}$ & $\tau$ & $\begin{array}{c}T_{\mathrm{ex}} \\
\mathrm{K}\end{array}$ & $\begin{array}{c}N \\
\mathrm{~cm}^{-2}\end{array}$ \\
\hline $\mathrm{ND}\left(0_{1}-1_{2}\right)$ & $-2.57 \pm 0.33$ & $0.74 \pm 0.14$ & $28.8 \pm 3.9$ & 4.6 & $(1.7 \pm 0.6) \times 10^{14}$ \\
\hline $\mathrm{ND}\left(0_{1}-1_{1}\right)$ & $-1.18 \pm 0.13$ & $0.82 \pm 0.14$ & $7.9 \pm 2.1$ & 4.5 & $(0.9 \pm 0.4) \times 10^{14}$ \\
\hline $\mathrm{NH}\left(0_{1}-1_{0}\right)$ & $-3.78 \pm 0.58$ & $0.60 \pm 0.08$ & $8.4 \pm 2.1$ & 9.5 & $(2.0 \pm 0.8) \times 10^{14}$ \\
\hline $\mathrm{NH}\left(0_{1}-1_{2}\right)$ & $-27.3 \pm 0.27$ & $0.64 \pm 0.08$ & $34.9 \pm 4.8$ & 7.7 & $(1.8 \pm 0.4) \times 10^{14}$ \\
\hline $\mathrm{NH}\left(0_{1}-1_{1}\right)$ & $-18.6 \pm 0.75$ & $0.63 \pm 0.08$ & $25.1 \pm 1.6$ & 8.8 & $(2.2 \pm 0.4) \times 10^{14}$ \\
\hline
\end{tabular}

Notes. $\tau$ is the total optical depth, $T_{\mathrm{ex}}$ the excitation temperature, $J_{v}\left(T_{\mathrm{ex}}\right)$ the radiation temperature at the frequency of the transition, $J_{v}\left(T_{\mathrm{bg}}\right)$ the radiation temperature of the cosmological background at $T_{\mathrm{bg}}=2.7 \mathrm{~K}, T_{\mathrm{c}}$ the brightness temperature of the continuum, $\Delta v$ the linewidth (full width at half maximum), and $N$ the total measured column density in the given species. $T_{\mathrm{ex}}$ was deduced from Col. (2). Because of uncertainties in some stages of the data reduction process, we estimate that the linewidths are not known to better than about $0.25 \mathrm{MHz}$. It is this uncertainty that we quote here in Col. (3).
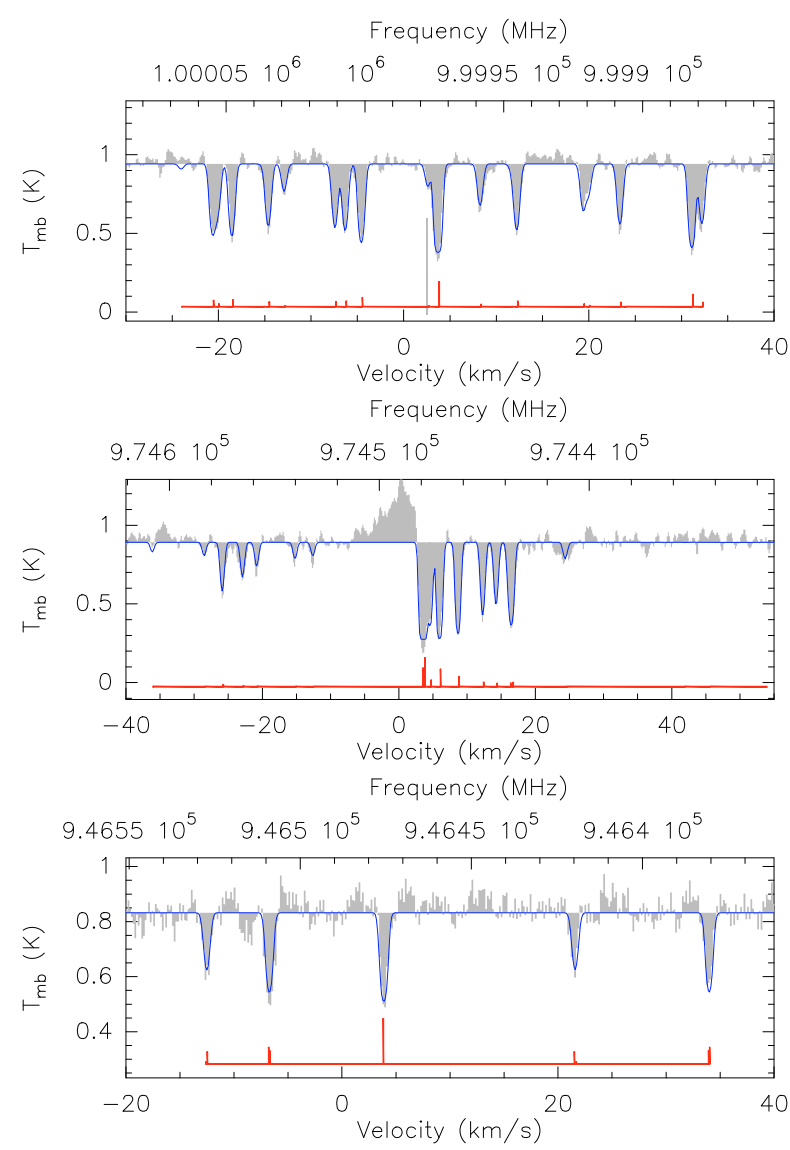

Fig. 2. $\mathrm{NH}\left(N_{J}=0_{1}-1_{0}\right)$ transition at $946.5 \mathrm{GHz}$ (top panel), $\mathrm{NH}\left(N_{J}=\right.$ $\left.0_{1}-1_{2}\right)$ transition at $974.5 \mathrm{GHz}$ (middle panel) and $\mathrm{NH}\left(N_{J}=0_{1}-1_{1}\right)$ transition at $1000.0 \mathrm{GHz}$ (bottom panel). The solid line fitting the spectra and the sketch at the bottom of each panel have the same signification as in Fig. 1. Note the HCN line towards $974.48 \mathrm{GHz}$.

possible for us to conclude anything about their relevance without a more complete chemical model.

We have reported the first detection of the radical ND by the high resolution heterodyne instrument HIFI on board the Herschel Space observatory, towards the young solar-mass protostar IRAS16293. The hyperfine structure is seen in absorption against the continuum background from the protostar, for both $\mathrm{ND}$ and $\mathrm{NH}$, which implies that the absorption arises from the cold envelope around the hot corino. The deuterium fractionation measured is very high, with a ratio $[\mathrm{ND}] /[\mathrm{NH}]$ between 30 and $100 \%$. Detailed radiative transfer and chemical modelling are needed to constrain the deuteration and formation of $\mathrm{NH}$ and this will be the subject of a future study.

Acknowledgements. HIFI has been designed and built by a consortium of institutes and university departments from across Europe, Canada and the United States under the leadership of SRON Netherlands Institute for Space Research, Groningen, The Netherlands and with major contributions from Germany, France and the US. Consortium members are: Canada: CSA, U.Waterloo; France: CESR, LAB, LERMA, IRAM; Germany: KOSMA, MPIfR, MPS; Ireland, NUI Maynooth; Italy: ASI, IFSI-INAF, Osservatorio Astrofisico di Arcetri-INAF; Netherlands: SRON, TUD; Poland: CAMK, CBK; Spain: Observatorio Astronómico Nacional (IGN), Centro de Astrobiología (CSIC-INTA). Sweden: Chalmers University of Technology - MC2, RSS \& GARD; Onsala Space Observatory; Swedish National Space Board, Stockholm University - Stockholm Observatory; Switzerland: ETH Zurich, FHNW; USA: Caltech, JPL, NHSC. We thank many funding agencies for financial support.

\section{References}

Bacmann, A., Lefloch, B., Ceccarelli, C., et al. 2003 ApJ, 585, L55 Caselli, P., Vastel, C., Ceccarelli, C., et al. 2008, A\&A, 492, 703

Caselli, P., van der Tak, F. F. S., Ceccarelli, C., \& Bacmann, A. 2003, A\&A, 403, 37

Ceccarelli, C., Bacmann, A., Boogert, A., et al. 2010, A\&A, 521, L22

Ceccarelli, C., Castets, A., Loinard, L., et al. 1998, A\&A, 338, 43

Comito, C., \& Schilke, P. 2002, A\&A, 395, 357

de Graauw, Th., Helmich, F. P., Phillips, T. G., et al. 2010, A\&A, 518, L6

Galloway, E. T., \& Herbst, E. 1989, A\&A, 211, 418

Klaus, Th., Takano, S., \& Winnewisser, G. 1997, A\&A, 322, L1

Linsky, J. L. 2007, Space Sci. Rev., 130, 367

Loinard, L., Castets, A., Ceccarelli, C., et al. 2002, P\&SS, 50, 1205

Marquette, J.-B., Rebrion, C., \& Rowe, B. R. 1988, J. Chem. Phys., 89, 2041

Molek, C. D., McLain, J. L., Poterya, V., \& Adams, N. G. 2007, J. Phys. Chem. A, 111, 6760

Müller, H. S. P., Thorwirth, S., Roth, D. A., \& Winnewisser, G. 2001, A\&A, 370, L49

Müller, H. S. P., Schlöder, F., Stutzki, J., \& Winnewisser, G. 2005, J. Mol. Struct., 742,215

Olofsson, A. O. H., Persson, C. M., Koning, N., et al. 2007, A\&A, 476, 791

Ott, S. 2010, in Astronomical Data Analysis Software and Systems XIX, ed. Y.

Mizumoto, K.-I. Morita, \& M. Ohishi, ASP Conf. Ser., in press

Parise, B., Ceccarelli, C., Tielens, A. G. G. M., et al. 2002, A\&A, 393, 49

Parise, B., Castets, A., Herbst, E., et al. 2004, A\&A, 416, 159

Pilbratt, G. L., Riedinger, J. R., Passvogel, T., et al. 2010, A\&A, 518, L1

Phillips, T. G., \& Vastel, C. 2003, in Chemistry as a Diagnostic of Star

Formation, ed. C. L. Curry, \& M. Fish [arXiv: astro-ph/0211610]

Roelfsema, P. R., Helmich, F. P., Teyssier, D., et al. 2010, A\&A, submitted

Roberts, H., Fuller, G. A., Millar, T. J., et al. 2007, A\&A, 381, 1026

Roberts, H., Herbst, E., \& Millar, T. J. 2003, ApJ, 591, L41

Roberts, H., Fuller, G. A., Millar, T. J., et al. 2002, A\&A, 381, 1026

Rodgers, S. D., \& Charnley, S. B. 2001, ApJ, 553, 613

Roueff, E., Lis, D. C., van der Tak, F. F. S., et al. 2005, A\&A, 438, 585

Saito, S., \& Goto, M. 1993, ApJ, 410, L53

Stark, R., Sandell, G., Beck, S. C., et al. 2004, ApJ, 608, 341

Talbi, D. 2009, J. Phys. Conf. Ser., 192, 012015

Thomas, R. D., Hellberg, F., Neau, A., et al. 2005, Phys. Rev. A, 71, 032711

van der Tak, F. F. S., Schilke, P., Müller, H. S. P., et al. 2002, A\&A, 388, L53

van Dishoeck, E. F., Blake, G. A., Jansen, D. J., \& Groesbeck, T. D. 1995, ApJ, 477, 760 
1 Laboratoire d'Astrophysique de Grenoble, UMR 5571-CNRS, Université Joseph Fourier, Grenoble, France

e-mail: aurore.bacmann@obs.ujf-grenoble.fr

2 Université de Bordeaux, Laboratoire d'Astrophysique de Bordeaux, Floirac, France

3 California Institute of Technology, Pasadena, USA

4 INAF - Istituto di Fisica dello Spazio Interplanetario, Roma, Italy

5 Infrared Processing and Analysis Center, Caltech, Pasadena, USA

${ }^{6}$ Centre d'Étude Spatiale des Rayonnements, Université Paul Sabatier, Toulouse, France

7 School of Physics and Astronomy, University of Leeds, Leeds, UK

8 LERMA and UMR 8112 du CNRS, Observatoire de Paris, Paris, France

9 Centro de Astrobiología, CSIC-INTA, Madrid, Spain

10 Max-Planck-Institut für Radioastronomie, Bonn, Germany

11 INAF Osservatorio Astrofisico di Arcetri, Florence, Italy

12 Astronomical Institute Anton Pannekoek, University of Amsterdam, Amsterdam, The Netherlands

13 Department of Astrophysics/IMAPP, Radboud University Nijmegen, Nijmegen, The Netherlands

14 IGN Observatorio Astronómico Nacional, Alcalá de Henares, Spain

15 Jet Propulsion Laboratory, Caltech, Pasadena, CA 91109, USA
16 SRON Netherlands Institute for Space Research, Groningen, The Netherlands

17 Ohio State University, Columbus, OH, USA

18 Harvard-Smithsonian Center for Astrophysics, Cambridge MA, USA

19 Johns Hopkins University, Baltimore MD, USA

20 Physikalisches Institut, Universität zu Köln, Köln, Germany

21 Institut de RadioAstronomie Millimétrique, Grenoble, France

${ }^{22}$ Leiden Observatory, Leiden University, Leiden, The Netherlands

23 Department of Physics and Astronomy, University College London, London, UK

${ }^{24}$ INAF - Osservatorio Astronomico di Roma, Monte Porzio Catone, Italy

25 Department of Astronomy, University of Michigan, Ann Arbor, USA

26 Max-Planck-Institut für Astronomie, Heidelberg, Germany

27 Kapteyn Astronomical Institute, University of Groningen, The Netherlands

${ }^{28}$ Laboratoire d'Études du Rayonnement et de la Matière en Astrophysique, UMR 8112 CNRS/INSU, OP, ENS, UPMC, UCP, Paris, France

29 CNRS/INSU, UMR 5187, Toulouse, France

30 CNRS/INSU, UMR 5804, Floirac Cedex, France

31 Institute of Astronomy, ETH Zürich, Zürich, Switzerland 